\title{
Neutrosophic Assignment Problem via BnB Algorithm
}

\author{
S. Krishna Prabha and S. Vimala
}

\begin{abstract}
This paper attempts to commence branch and bound technique to unravel the triangular fuzzy neutrosophic assignment problem (TFNAP). So far there are many researches based on fuzzy and intuitionistic fuzzy assignment problems; this is the first paper to deal with TFNAP which have been introduced as a simplification of crisp sets and intuitionistic fuzzy sets to indicate vague, imperfect, unsure, and incoherent notification about the existent world problem. Here a real-life agricultural problem where the farmer's objective is to locate the optimal assignment of paddocks to crops in such comportment that the total fertilizer cost becomes least is worked out to illustrate the efficiency of the branch and bound $(\mathrm{BnB})$ algorithm in neutroshopic approach.
\end{abstract}

Keywords Triangular fuzzy neutroshopic assignment problem · Agricultural problem $\cdot$ Branch and bound algorithm

\section{Introduction}

To simplify the idea of fuzzy sets and intuitionistic fuzzy sets, Smarandache in 1998 [7] projected the perception of neutrosophic set and neutrosophic logic for managing problems concerning vague, imperfect, uncertain, and incoherent information which may not be handled using fuzzy sets and intuitionistic fuzzy sets. Three different membership degrees explicitly truth-membership degree (T), indeterminacy-membership degree (I), and falsity-membership degree $(\mathrm{F})$, which

\footnotetext{
S. Krishna Prabha $(\bowtie)$

Department of Mathematics, Mother Teresa Women's Universiy, Kodaikannal, India

Department of Mathematics, PSNA College of Engineering and Technology, Dindigul, India e-mail: jvprbh1@gmail.com; krishna_praba@psnacet.edu.in.

S. Vimala

Department of Mathematics, Mother Teresa Women's Universiy, Kodaikannal, India

e-mail: tvimss@gmail.com
} 
elongate out flanked through nonstandard unit interval $]-0,1+[$, are the categorization of the perception of neutrosophic set. Smarandache [7] and Wang et al. projected a subclass of the neutrosophic sets named single-valued neutrosophic sets (SVNS). By merging triangular fuzzy numbers (TFNs) and single-valued neutrosophic set (SVNS), Biswas et al. [4] introduced the idea of triangular fuzzy neutrosophic sets (TFNS).Trapezoidal fuzzy neutrosophic set was proposed by Ye [17], and he urbanized weighted arithmetic and geometric averaging for TFNS. Assignment problem (AP) is applied universal in unraveling genuine real tribulations. Among the premeditated optimization tribulations in administration discipline, the assignment problem has been widely enforced in both mechanized and repair systems. The intent of assignment problem is to consign $n$ tasks to $n$ machines at a least cost. As conservative traditional assignment problems cannot be effectively dealt with situations concerning imprecision in the data, the concept of fuzziness proposed by Zadeh [18] is employed. Researchers like Chen [6], Chen Liang-Hsuan et al. [5], and Long-Sheng Huang et al. [10] have explored various concepts for solving assignment problems. Ones assignment method for unraveling assignment problems was put forth by Hadi Basirzadeh [8]. Yager [16] has introduced a new method to rank the fuzzy subsets of unit interval. Various ranking methods have been proposed by Abbasbandy et al. [1] and Nagarajan et al. [12] to defuzzify fuzzy numbers. Srinivas and Ganesan [14] have applied branch and bound method for unraveling assignment problems. Transportation problems under neutrosophic domain were resolved by Thamaraiselvi et al. [15] and Akansha Singh et al. [2]. With linguistic variables, Anil and Khot [3] resolved fuzzy assignment problem through $\mathrm{BnB}$ method. Broumi et al. [13] have discussed the shortest path problem in neutrosophic domain. An application in agriculture by intuitionistic fuzzy assignment problem has been derived by Lone et al. [9]. Aggravated by the works done by the above researchers, in this paper branch and bound method is projected for solving triangular neutroshopic fuzzy assignment problems (TFNAP).

\section{Preliminaries}

Some important results regarding neutrosophic sets, single-valued neutrosophic sets, and triangular fuzzy neutrosophic sets have been referred from [13, 17].

The concept of score function $\mathrm{S}$ and accuracy function $\mathrm{H}$ are proposed by Ye[17].

Definition 2.1 Let $F[0,1]$ be the set of all triangular fuzzy numbers on $[0,1]$ and $\mathrm{X}$ be a finite universe of discourse. A triangular fuzzy neutrosophic set (TFNS) in X is given by $\tilde{A}=\left\{\left\langle x: \tilde{T}_{A}(x), \tilde{I}_{A}(x), \tilde{F}_{A}(x)\right\rangle, x \in X\right\}$ where $\tilde{T}_{A}(x): X \longrightarrow F[0,1]$, $\tilde{I}_{A}(x): X \longrightarrow F[0,1]$ and $\tilde{F}_{A}(x): X \longrightarrow F[0,1]$,

The triangular fuzzy numbers $\tilde{T}_{A}(x)=\left(T_{A}^{1}(X), T_{A}^{2}(X), T_{A}^{3}(X)\right)$,

$\tilde{I}_{A}(x)=\left(I_{A}^{1}(X), I_{A}^{2}(X), I_{A}^{3}(X)\right)$,

$\tilde{F}_{A}(x)=\left(F_{A}^{1}(X), F_{A}^{2}(X), F_{A}^{3}(X)\right)$, 
respectively, denote the truth-membership, indeterminacy- membership, and falsitymembership degree of $x$ in $\tilde{\mathrm{A}}$

and for every $x \in X, 0 \leq T_{A}^{3}(X)+I_{A}^{3}(X)+F_{A}^{3}(X) \leq 3$.

For notational convinence the triangular fuzzy neutrosophic value TFNV is denoted by $\left(T_{A}^{1}(X), T_{A}^{2}(X), T_{A}^{3}(X)\right)=(a, b, c)$,

$\left(I_{A}^{1}(X), I_{A}^{2}(X), I_{A}^{3}(X)\right)=(e, f, g),\left(F_{A}^{1}(X), F_{A}^{2}(X), F_{A}^{3}(X)\right)=(r, s, t)$

Definition 2.2 Let $\tilde{A}=\langle(a, b, c),(e, f, g),(r, s, t)\rangle$ be a TFNV; then score function $S\left(\tilde{A}_{1}\right)$ and accuracy function $H\left(\tilde{A}_{1}\right)$ are defined as follows:

$S\left(\tilde{A}_{1}\right)=\frac{1}{12}\left[8+\left(a_{1}+2 b_{1}+c_{1}\right)-\left(e_{1}+2 f_{1}+g_{1}\right)-\left(r_{1}+2 s_{1}+t_{1}\right)\right]$

$H\left(\tilde{A}_{1}\right)=\frac{1}{4}\left[\left(a_{1}+2 b_{1}+c_{1}\right)-\left(r_{1}+2 s_{1}+t_{1}\right)\right]$

\section{Assignment Method [6, 8, 9, 11, 12]}

Mathematically a TFNAP is formulated as minimize $\mathrm{z}=\sum_{i=1}^{n} \sum_{j=1}^{n}\left(\tilde{C}_{i j}\right)^{I}$ where $i=1,2,3, ., ., ., . n, j=1,2,3, ., ., ., . n$

Subject to $\sum_{i=1}^{n} x_{i j}=1, i=1,2,3 \ldots . . n$

$\sum_{j=1}^{n} x_{i j}=1, j=1,2,3 \ldots n, x_{i j} \in\{0,1\}$

where $x_{i j}=\left\{\begin{array}{c}1 \text { ifthei }{ }^{\text {th }} \text { crop is assigned to thej }{ }^{\text {th }} \text { paddock } \\ 0 \text { otherwise }\end{array}\right.$

$\tilde{C}_{i j}=\left(\left(C_{i j}^{1}, C_{i j}^{2} c_{i j}^{3}\right)\left(C_{i j}^{1^{I}}, C_{i j}^{2^{I}}, C_{i j}^{3^{I}}\right)\right)$

$\tilde{C}_{i j}^{I}$ is the cost of allotting the $i$ th crop to the $j$ th paddock. The goal is to reduce the total cost of allotting all crops to the paddocks (One crop to one paddock). If the costs of $\tilde{c}_{i} j^{I}$ are TNF costs, then the TFNAP becomes

$Y(\tilde{Z})=\sum_{i=1}^{n} \sum_{j=1}^{n} Y\left(\tilde{C}_{i j}\right)^{I} x_{i j}$

subject to the same conditions.

For an unbalanced problem, add dummy rows/ columns and then follow the same procedure.

\section{Branch and Bound Technique $[3,11,14]$}

1. Presume that the source node is 0 by taking the level number as $\delta$ and allotment number as $\beta$ in the present node of a branching tree.

2. $\mathbb{I}_{\beta}^{\delta}$ be an allotment at level $\delta$ of the branching tree. The set of assigned cells be $\S_{A}$ up to the node $\mathbb{I}_{\beta}^{\delta}$ as of the root node (set of $\mathrm{i}, \mathrm{j}$ values regarding the alloted cells up to the node as of the source node), assuming the upper bound of the partial allotment up to $\|_{\beta}^{\delta}$ be $V_{\epsilon}$ such that $V_{\epsilon}=\Sigma_{i, j \in X} C_{i j}+\Sigma_{i \in x} \Sigma_{j \in y}^{\prime}$ $\max C_{i j}$ 
The cell entry of the profit matrix with respect to the 1th row and $j$ th column is denoted as $C_{i j}$. Presume $\mathrm{X}$ as the set of rows that are not eliminated up to the node $\mathbb{I}_{\beta}^{\delta}$ from the node in the branching node.

\subsection{Branching Methodology}

1. The column noted as $\delta$ of the AP will be allotted with the prime row of the AP at level $\delta$.

2. The end node at the upmost level is to be taken into account for further branching, if there is a tie on the upper bound.

3. The optimality is obtained only if the greatest upper bound occurs to be at any one of the end nodes at the (n-1)th level. The optimal solution will be the allotment on the trail from the source node together with the omitted pair of row/column combination.

\section{Numerical Example}

Consider a TFNAP, where a farmer intends to plant four disparate crops in each of four equal-sized paddocks with rows instead of four different crops $\mathrm{C} 1, \mathrm{C} 2$, C3, C4 and columns instead of four equal sized paddocks like P1, P2, P3 and $\mathrm{P} 4$. The nutrient requirements required for different crops vary, and the paddocks vary in soil fertility. Thus the cost of the fertilizers which must be applied depends on which crop is grown in which paddock. The cost matrix be $\left[\tilde{C}_{i j}\right]$ whose components are given as TFNS. The farmer's aim is to locate the best allotment of paddocks to crops in such a manner that the entire fertilizer price becomes least (Table 1).

By using score function formula, TFNSV are converted to crisp values as follows:

$$
\begin{aligned}
S\left(\tilde{A}_{1}\right) & =\frac{1}{12}\left[8+\left(a_{1}+2 b_{1}+c_{1}\right)-\left(e_{1}+2 f_{1}+g_{1}\right)-\left(r_{1}+2 s_{1}+t_{1}\right)\right] \\
\tilde{C}_{11} & =0.25, \tilde{C}_{12}=0.33, \tilde{C}_{13}=1.33, \tilde{C}_{14}=3.33, \\
\tilde{C}_{21} & =0.33, \tilde{C}_{22}=0.083, \tilde{C}_{23}=1.58, \tilde{C}_{24}=2.92, \\
\tilde{C}_{31} & =1.67, \tilde{C}_{32}=3.33, \tilde{C}_{33}=0.583, \tilde{C}_{34}=0.583, \\
\tilde{C}_{41} & =2.92, \tilde{C}_{42}=0.33, \tilde{C}_{43}=0.583, \tilde{C}_{44}=0.583,
\end{aligned}
$$

$$
\left(\begin{array}{cccc}
0.25 & 0.33 & 1.33 & 3.33 \\
0.33 & 0.083 & 1.58 & 2.92 \\
1.67 & 3.33 & 0.083 & 0.25 \\
2.92 & 0.33 & 0.583 & 0.583
\end{array}\right)
$$




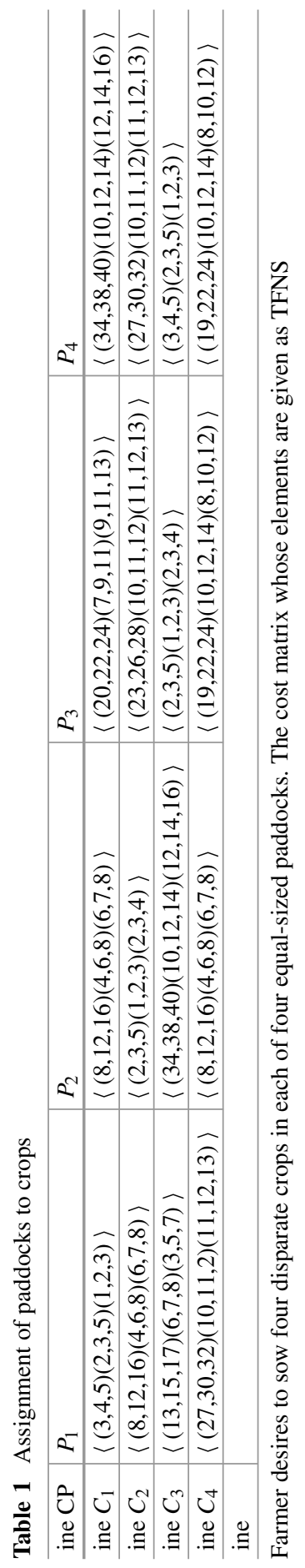


At first no crops are allotted to any paddocks, so the allotment $\sigma$ at the source (level 0 ) of the branching tree is the empty set, and the subsequent lower bound is also 0 for all. $P_{11}^{I}=0.25+[2.92+3.33+0.583]=7.083$,

$$
\begin{aligned}
& P_{21}^{I}=0.33+[3.33+3.33+0.583]=7.573, \\
& P_{31}^{I}=1.67+[3.33+2.92+0.583]=8.503, \\
& P_{41}^{I}=2.92+[3.33+1.58+3.33]=11.16,
\end{aligned}
$$

Further branching is done from the terminal node which has the greatest upper bound. $P_{11}^{I}, P_{21}^{I}, P_{31}^{I}$, and $P_{41}^{I}$ are the terminal nodes. The node $P_{41}^{I}$ has the greatest upper bound .Eliminate fourth row and first column. Hence further branching from this node is shown as follows:

$V_{22}=C_{41}+C_{12}+\Sigma_{i \in x} \Sigma_{j \in y} \max C_{i j}$

$$
\left(\begin{array}{ccc}
0.33 & 1.33 & 3.33 \\
0.083 & 1.58 & 2.92 \\
3.33 & 0.083 & 0.25
\end{array}\right)
$$

$P_{12}^{I}=0.33+[2.92+0.25]=3.5, P_{22}^{I}=0.083+[3.33+0.25]=3.663$, $P_{32}^{I}=3.33+[3.33+2.92]=9.58$,

At this stage the nodes $P_{41}^{I}, P_{12}^{I}, P_{22}^{I}$, and $P_{32}^{I}$ are the terminal nodes. Among these nodes $P_{32}^{I}$ is the upper bound. By considering end nodes at the uppermost for further branching, eliminate 3 rd row and 2 nd column. $V_{33}=C_{41}+C_{32}+\Sigma_{i \in 4} \Sigma_{j \in 4} \max C_{i j}$

$$
\left(\begin{array}{ll}
1.33 & 3.33 \\
1.58 & 2.92
\end{array}\right)
$$

$P_{13}^{I}=[1.33+2.92]=4.25, P_{23}^{I}=[1.58+3.33]=4.91$; eliminate 2 nd row and 3rd column. $P_{41}^{I}=3.33$. The optimal Assignment is given by $P_{14}^{I}+P_{32}^{I}+P_{23}^{I}+P_{41}^{I}=$ $3.33+1.58+3.33+2.92=11.16$ (Fig. 1$)$.

\section{Conclusion}

The assignment cost has been measured as vague numbers narrated by TFNS in this manuscript. The TFNAP has been defuzzified into crisp AP by score value, and $\mathrm{BnB}$ technique has been implied to derive an optimal result for the first time in neutroshopic assignment problems. Mathematical instance has been exposed that the allocation acquired is best. The optimal assignment of paddocks to crops is found in such a manner to satisfy the farmer's objective of making the total fertilizer cost becomes minimum. In the future the problem can be unraveled by the following methods: reduced matrix method, ones assignment method, approximation method, and best candidate method. BnB technique can be applied in solving integer programming, nearest neighbor search, knapsack problem, bin packing, and MAXSAT problems. 


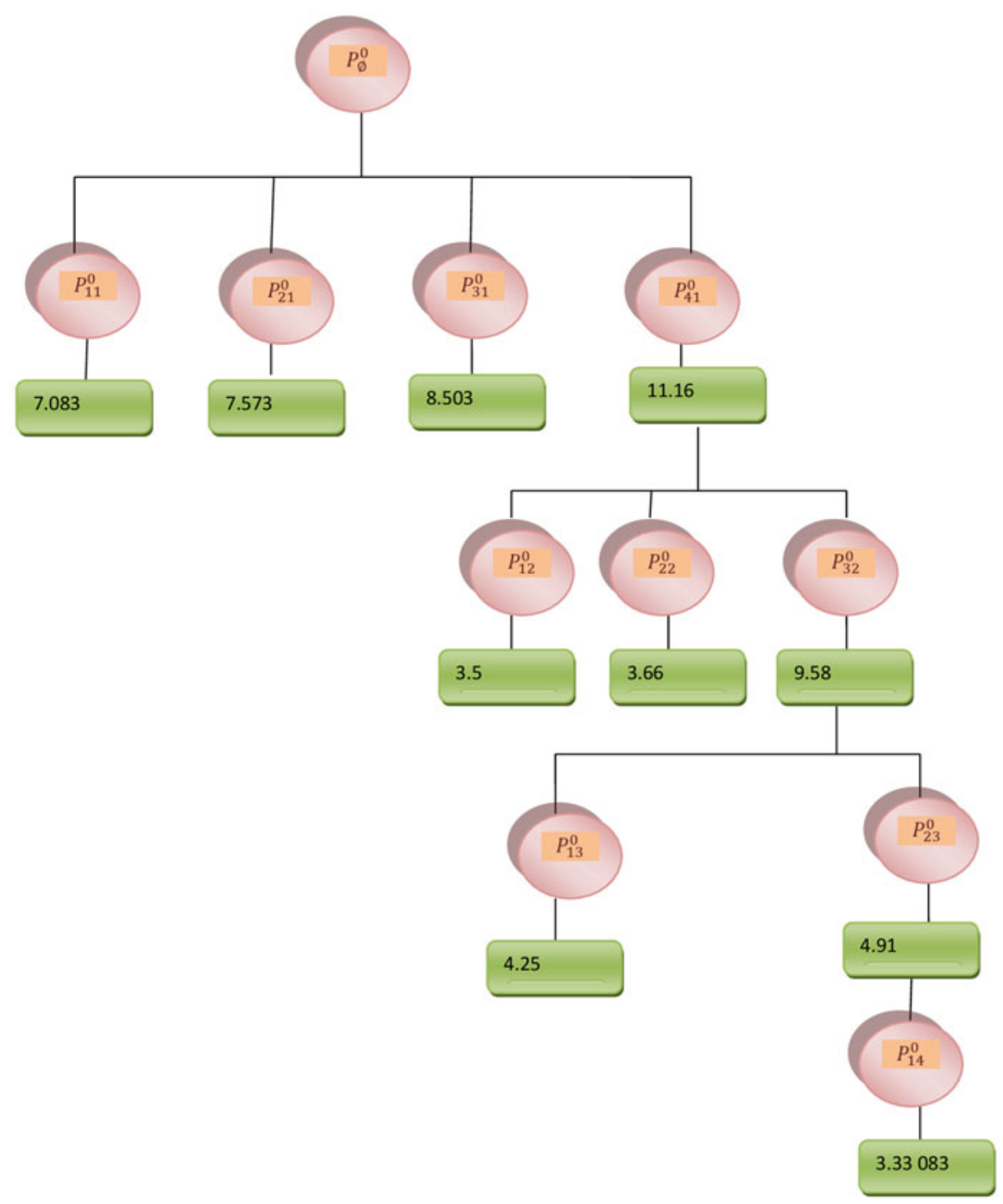

Fig. 1 Optimal solution for type-4 triangular neutroshopic assignment problem

\section{References}

1. Abbasbandy,S.,Hajjari,T.: A new approach for ranking of trapezoidal fuzzy numbers. Computers and Mathematics with Applications. 57,413-419(2009)

2. Akansha Singh,Amit Kumar, Apppadoo,S.S.: Modified approach for optimization of real life transportation problem in neutrosophic environment.Mathematical Problems in Engineering.(2017) Article id: 2139791

3. Anil Gotmare,D., Khot, P.G.: Solution of fuzzy assignment problem by using branch and bound technique with application of lingustic variable.International Journals of Computer and Technology.15(4) (2016) 
4. Biswas,P. Pramanik,S. Giri,B.C.: Aggregation of triangular fuzzy neutrosophic set information and its application to multi attribute decision making.Neutrosophic Sets and Systems.12,20 40(2016)

5. Chen Liang-Hsuan, Lu Hai-Wen.: An extended assignment problem considering multiple and outputs.Applied Mathematical Modelling.31,2239-2248(2007)

6. Chen, M.S.: On a fuzzy assignment problem.Tamkang Journal.22,407-411(1985)

7. Florentin Smarandache.: Neutrosophic ,neutrosophic probability set and logic.Amer.Res Press .Rehoboth.USA.105p,(1998)

8. Hadi Basirzadeh.: Ones assignment method for solving assignment problems.Applied Mathematical Sciences.6(47), 2345-2355(2012)

9. Lone,M.A.,Mir,S.A.,Ismail,Y., Majid,R.: Intustinistic fuzy assignment problem, an application in agriculture .Asian Journal of Agricultural Extension,Economics and Socialogy.15(4), 16(2017)

10. Long-Sheng Huang., Li-pu Zhang.: Solution method for fuzzy assignment problem with restriction of qualification.Proceedings of the Sixth International Conference on Intelligent Systems Design and Applications. ISDA'06 (2006)

11. Muruganandam,S.,Hema,K.: Solving fully fuzzy assignment problem using branch and bound technique.Global Journal of Pure and Applied Mathematics.13(9), 4515-4522(2017)

12. Nagarajan,R.Solairaju,A.:Assignment problems with fuzzy costs under robust ranking techniques.International Journal of Computer Applications.6,(2010)

13. Said Broumi, Assia Bakali, Mohemed Talea, Florentien Smarandache .: Shortest path problem under triangular fuzzy neutrosophic information. 10th International Conference on Software, Knowledge, Information Management and Applications (SKIMA). https://doi.org/978-15090-3298-3/16@2016 IEEE(2016)

14. Srinivas,B. Ganesan,G.: Method for solving branch-and-bound technique for assignment problems using triangular and trapezoidal fuzzy numbers.International Journal In Management And Social Science.3(3),(2015)

15. Thamaraiselvi,A. Shanthi ,R.: A new approach for optimization of real life transportation problem in neutrosophic environment.Mathematical Problems in Engineering.(2016) doi: 5950747.

16. Yager,R,R.: A procedure for ordering fuzzy subsets of the unit interval.Information Sciences.vol. 24(2), 143-161 (1981)

17. Ye,J.: Trapezoidal fuzzy neutrosophic set and its application to multiple attribute decision making.Soft Computing.(2015) https://doi.org/10.1007/s00500-015-1818-y

18. Zadeh, L.: Fuzzy sets, Information and Control.8(3),338-353 (1965) 